\title{
Le suffixe adjectival -ble : Impacts sémantique et syntaxique de la dérivation
}

Martine Schuwer

\section{CpenEdition}

\section{Journals}

Édition électronique

URL : http://journals.openedition.org/asp/2315

DOI : 10.4000/asp. 2315

ISBN : 978-2-8218-0380-0

ISSN : 2108-6354

Éditeur

Groupe d'étude et de recherche en anglais de spécialité

Édition imprimée

Date de publication : 1 décembre 1999

Pagination : $29-54$

ISSN : 1246-8185

Référence électronique

Martine Schuwer, "Le suffixe adjectival -ble : Impacts sémantique et syntaxique de la dérivation », ASp [En ligne], 23-26 | 1999, mis en ligne le 05 mars 2011, consulté le 19 avril 2019. URL : http:// journals.openedition.org/asp/2315; DOI : 10.4000/asp.2315

Ce document a été généré automatiquement le 19 avril 2019

Tous droits réservés 


\title{
Le suffixe adjectival -ble: Impacts sémantique et syntaxique de la dérivation
}

\author{
Martine Schuwer
}

1 Dans le cadre général d'une recherche sur la syntaxe de l'adjectif en anglais, la présente étude porte sur les adjectifs en -ble dont la syntaxe est réputée contrainte, certains ne pouvant figurer qu'en épithète, d'autres seulement en position attributive.

2 Strictement parlant, -ble est considéré comme un suffixe lorsqu'il participe de la formation d'un dérivé à partir d'une base existante en langue, ou comme une terminaison dans le cas des adjectifs empruntés (la racine appartient alors à la langue d'origine de l'emprunt). Il ne sera pas fait ici de distinction entre les deux modes de formation, compte tenu du fait que, directement ou non, ce morphème est toujours la trace d'une dérivation, car ce procédé lexicogénique, emprunté certes, s'est trouvé ré-approprié dans la langue anglaise.

3 En raison de ce choix méthodologique, c'est-à-dire la sélection d'un trait morphologique comme critère de constitution du corpus, on pose implicitement que la dérivation, en tant que telle, peut constituer un élément soit partiellement déclencheur, soit révélateur de l'emploi syntaxique. Mais tous les dérivés en -ble ne sont pas syntaxiquement contraints, ce n'est que lorsque certaines conditions sont réunies que l'adjectif occupe une place assignée.

4 En effet, comme on l'a constaté lors d'études menées sur d'autres dérivés adjectivaux ${ }^{1}$, le comportement syntaxique est étroitement lié :

- au type de base susceptible d'être suffixée : les modèles de dérivation, quand bien même l'étude diachronique montre qu'ils évoluent (certains devenant obsolètes, par exemple), suivent des schémas bien définis;

- aux propriétés du référent que l'adjectif dérivé modifie (qu'il soit animé humain ou non, qu'il s'agisse d'un prédicat nominalisé...);

- à la nature de la relation que le suffixe établit entre la base dérivée et le nom-support. 
5 Ainsi, partant des formes suffixées, en étudiant la nature de la base et le type de référent qualifié, il est possible d'établir les conditions d'apparition des contraintes, et d'évaluer l'impact de la suffixation. L'objectif est de mesurer les effets de l'interaction - rendue possible ou déclenchée par la suffixation - entre les propriétés attachées aux référents de la base et du substantif qualifié.

6 La première partie sera consacrée à quelques remarques générales sur l'évolution et la place de ce suffixe dans la langue anglaise.

\section{Remarques générales}

7 Le morphème -ble constitue le suffixe d'adjectifs dérivés soit de verbes - le plus souvent transitifs, mais aussi intransitifs (variable) - soit de noms (fashionable, reasonable).

\section{1. Étymologie du suffixe}

8 L'anglais doit ce suffixe à l'ancien français qui l'a lui-même emprunté au latin; il permettait la formation d'adjectifs déverbaux et dénominaux ${ }^{2}$.

9 À -ble correspondent trois variantes, l'adjectif latin pouvant être dérivé soit sur la base, soit sur la racine participiale d'un verbe :

10 -uble, du latin -ubilis, suffixant la base des verbes en -vere (solvere, volvere), représenté dans deux adjectifs seulement (soluble, voluble)

11 -ible, du latin -ibilis, suffixant la base ou la racine participiale des verbes en -ere (possere, terrere, videre), présent dans de nombreux adjectifs empruntés (impossible, terrible), mais ne constituant pas un suffixe productif ${ }^{3}$

12 -able de -abilis, pouvant suffixer soit les verbes en -are (arare>arabilis), puis en -ere ( capere>capabilis), soit des substantifs (amicus>amicabilis). Seule cette variante est productive en anglais, et presque toujours sur une base verbale.

13 L'intégration de ce modèle de suffixation a connu plusieurs étapes. C'est tout d'abord sous la forme d'emprunts au vieux français qu'il est apparu en moyen anglais: feeble (XII), noble (XIII). C'est seulement au début du XIVe que -ble commença sans doute à être perçu comme un suffixe, car de très nombreux adjectifs sont alors empruntés (reasonable, seasonable...). Enfin, vers la fin du XIV e siècle, -ble acquiert le statut de suffixe productif, car il dérive des adjectifs à partir de bases anglaises. Il est fort probable que la similitude du suffixe et de l'adjectif able (de habilis dérivé de habere), qui sont étymologiquement distincts, a considérablement accéléré l'intégration de cet emprunt (une des plus rapides).

\subsection{Types de base}

\subsubsection{Origine linguistique}

14 Les adjectifs en -ble peuvent avoir été empruntés ou résulter de créations, selon plusieurs procédés de formation.

Emprunts 
L'anglais a directement emprunté, parfois au latin, des adjectifs en -bilis (inevitable XV), mais le plus souvent au français, des formes en -able ou en -ible : cultivable (1682), eligible (1561), honourable (XIV), incapable (1601), miserable (XVI), movable (1430), responsible (1643), returnable (1658), sensible (XIV), veritable (1474).

\section{Créations}

L'anglais a créé de nombreux adjectifs en -able suivant des modes de composition diversifiés.

base germanique, verbale ou nominale :

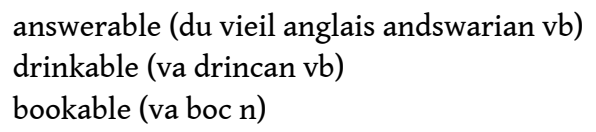

À la racine germanique peuvent aussi être associés des affixes empruntés : renewable (repréfixe latin, new du v-a ne(o)we).

base romane : disposable (1943 dans son sens actuel, issu de dispose vb, de l'ancien français disposer)

La racine romane peut également être précédée d'un préfixe germanique: unavailable (XIX), dont la formation est incertaine ; il est considéré soit comme dérivé par préfixation de available, soit comme issu de unavail, par dérivation inverse de unavailing. Quelle que soit l'hypothèse retenue, il s'agit d'une formation hybride, constituée d'une part d'un préfixe germanique (un-), d'une base et d'un suffixe empruntés d'autre part.

20 L'anglais a également créé des adjectifs en -ble suivant les règles de formation de la base d'origine : perfectible (1635) (qui, selon Marchand (1969: 177), n'existait pas en latin) dérivé de perfect $v b$, est effectivement formé à partir du participe passé du verbe latin perficere (formations latines similaires visible, formé sur vis- racine participiale de videre). Ce cas est rare.

21 C'est à son origine étrangère que -ble doit son adaptabilité (Marchand 1969 : 175), et ceci explique la diversité des modes de formation de ces dérivaux adjectivaux.

\subsubsection{Nature de la base}

Il existe en anglais quelques adjectifs dénominaux en -ble, mais ce mode de dérivation n'a jamais été très productif. C'est sans doute l'emprunt au français de formes dénominales ( honourable, veritable) qui a permis ou suscité la création de actionable (1591), conscionable (1549, accordant with good conscience, just), marriageable (1555), treasonable (1375).

En anglais contemporain, les dérivés sont majoritairement issus de verbes transitifs (directs ou indirects), plus rarement de verbes intransitifs : en moyen anglais, suivant le modèle latin ou français, a ainsi été emprunté durable, et forgé variable.

Aussi les adjectifs en -ble dérivés de noms ou de verbes intransitifs ne constituent-ils qu'une proportion limitée du lexique anglais contemporain.

La dérivation par préfixation a toujours été active, voire la dérivation inverse (par suppression du préfixe), la forme préfixée pouvant même exister préalablement à la forme non préfixée: unspeakable (1400)/speakable (1483), unavoidable (1577)/avoidable (1683). 
26 Ainsi, en latin, comme en français et en anglais, -ble peut suffixer des adjectifs dénominaux ou déverbaux. S'il est parfois difficile de déterminer avec certitude l'origine de la base ${ }^{4}$, il semble que les adjectifs déverbaux aient toujours été de loin les plus nombreux, et ce procédé de dérivation est le plus productif en anglais contemporain. L'analyse de récentes formations néologiques en $-b l e^{5}$ illustre la stabilité en diachronie des modes de dérivation. Sur 12 adjectifs en effet, un seul est dénominal (wifeable). Parmi les déverbaux :

- 1 est dérivé, par double affixation, d'un verbe ergatif : unmeltable

- 7 sont dérivés, par préfixation, de verbes transitifs ou complexes: inexorcizable, nonclassifiable, rewritable, unfilmable, unsponsorable, unspendable, unputdownable

- 2 sont dérivés de verbes transitifs : abusable, hissable

- 2 sont issus d'emprunts déverbaux dérivés par préfixation : super-flexible, semi-admirable

\subsection{Apport sémantique du suffixe}

Selon l'origine et la nature de la base, ainsi que la date d'apparition dans la langue, les adjectifs en -ble se prêtent à une interprétation active, ou passive, parfois les deux, l'ambiguïté étant levée par le contexte, plus précisément par le sémantisme du nomsupport.

Pour les formations actuelles, on explique généralement, comme pour rewritable par exemple, qu'un adjectif en -ble peut être paraphrasé par un schéma du type that can be Ved . Cette glose permet de constater que -ble est la trace de deux opérations : l'une de l'ordre de la modalisation (can), l'autre portant sur le choix du terme de départ de la relation ici le but - et ayant pour effet la focalisation sur le substantif ainsi qualifié, et dont témoigne l'emploi de la voix passive dans la glose. Ce schéma paraphrastique correspond à ce qu'on appelle parfois le « sens passif » de certains adjectifs en -ble.

30 Cependant, pour quelques formations anciennes citées par Jespersen et Marchand, ce schéma paraphrastique n'est pas applicable, certains adjectifs ${ }^{6}$ ayant, pour reprendre leurs termes, un sens "actif». Cette dénomination est ambiguë. Seule une analyse des gloses de ces adjectifs permet de rendre compte du rapport établi par -ble entre les noms qualifiés et ces dérivés anciens. On relève trois cas de figure.

31 Les dénominaux peuvent le plus souvent être paraphrasés en that has $N$ (that has knowledge ${ }^{7)}$ :

limousines with knowledgeable chauffeurs-guides ${ }^{8}$

N1-able N2

ou bien, dans le cas où N2 n'est pas un animé humain, en that has the characteristics of ( comfort, force):

He expressed his views in very forcible words ${ }^{9}$

N1-ble N2

Have signale que le suffixe -ble opère un repérage à valeur de différenciation entre le syntagme nominal (chauffeurs-guides/words) et le nom (knowledge/force) dont est dérivé l'adjectif.

Quant à la plupart des déverbaux, ils sont paraphrasables en that Vs (that suits, perishes, shrinks, varies, deceives) : 
the variable nature of the English climate

a film suitable for young children

Le corpus a été constitué à partir des indications dictionnairiques figurant dans Longman Dictionary of Contemporary English (LDOCE), et Collins Cobuild (CC), dictionnaires dont on sait que la nomenclature a été établie à partir de corpus écrits et oraux, et ainsi fondée sur des

emplois authentiques.

[1] There are passable maps and better summary notes on each territory.

Geographic Journal. Cité par Webster's

[2] Many of these roads are not passable in bad weather. CC

[3] changeable : given or prone to change = capricious.

He was as changeable as the weather. CC

[4] a provision changeable at will. Webster's

On constate ainsi que ce même suffixe peut être porteur de trois opérations différentes, selon l'ancienneté de la formation d'une part, et le type de base d'autre part.

L'anglais a bien entendu hérité du latin et du français la variété de ces valeurs. Ce suffixe est par conséquent remarquable à plusieurs égards : plus qu'un indicateur de catégorie grammaticale (par contraste avec d'autres suffixes adjectivaux, tels -ic, -ical, -an...), il permet de créer des adjectifs dénominaux et déverbaux; en tant que morphème emprunté, il peut être associé à une base germanique ou romane, enfin, lorsqu'il suffixe une base verbale, il est susceptible d'établir plusieurs types de relation entre l'adjectif dérivé et le syntagme nominal qualifié.

séparable dans la mesure où il est « susceptible d'être perçu comme distinct du reste du mot » (Guierre 1979 : 358). Cependant, notamment dans le cas des emprunts, la structure interne de l'adjectif est opaque et perd toute motivation morphologique. C'est en ce sens que certains adjectifs peuvent être considérés comme " dérivés de pseudo-morphèmes » (Duchet \& Fournier 1989), ce qui modifie le statut du suffixe qui constituerait alors un morphème non séparable étant donné que le contenu sémantique de l'adjectif ne peut se déduire à coup sûr directement de celui du radical et du suffixe ${ }^{10}$.

\subsection{Les adjectifs syntaxiquement contraints en -ble}


41 Aux formes recensées comme syntaxiquement contraintes, on a inclus des adjectifs dont seuls les exemples d'emploi témoignent d'une syntaxe obligée. La recherche d'occurrences en contexte a confirmé le bien-fondé de ces ajouts.

variante orthographique Son emploi est strictement prénominal, alors qu'en français, véritable peut - non indifféremment - figurer avant ou après le substantif qualifié : une véritable catastrophe/de l'or véritable. On constate que le sens actuel de veritable correspond à l'emploi obligatoirement prénominal en français. Cet adjectif est authentiquement dénominal, dérivé sur veritas (formation similaire amicus > amicabilis), et ainsi suffixé, correspond au schéma paraphrastique : that has the characteristics of N2. Marriageable et treasonable sont peu usités, $\mathrm{BNC}^{13}$ n'en offre respectivement que 26 et 32 occurrences ${ }^{14}$. Bien qu'ils ne soient pas officiellement recensés comme syntaxiquement contraints, leurs conditions d'emploi dénotent une forte propension à la position prénominale.. marriageable (1555 
marriage $\mathrm{N}+-a b l e$ ), "fit for marriage », a supplanté marriable (moyen anglais, du français mariable). Selon OED, le français mariage aurait été perçu comme verbe, et par voie de conséquence marriageable conçu comme la dérivation appropriée, conformément au procédé de dérivation le plus courant. Il est étonnant que le dérivé «bien formé » n'ait pas finalement survécu.

51 Ainsi, malgré son origine nominale, et peut-être également en raison des confusions évoquées ci-dessus, marriageable est tout à fait assimilable à un adjectif déverbal: qualifiant un animé humain (marriageable daughters), il évoque un procès (marry) relatif à celui-ci (daughters); dans les autres cas, l'adjectif délivre une information de type adverbial (marriageable age: when $X$ can marry) et il s'agit alors d'une hypallage... treasonable (1375), non susceptible de qualifier un animé humain ${ }^{15}$, signifie, selon OED, « of the nature of treason", s'avère le plus souvent associé à des prédicats nominalisés (act, attempt, communication). Si tel n'est pas le cas, la qualification est de nature adverbiale : treasonable times. Lorsque l'information véhiculée par l'adjectif est de type adverbial, la nature circonstancielle de la qualification interdit toute prédication directe en be. Dans les autres cas, la prédication est possible mais rare, et la suite adjectif+nom peut être considérée comme résultant d'une validation antérieure.

\subsubsection{Impact de la suffixation} nom dont est dérivé l'adjectif? Les relations susceptibles d'être instaurées entre N1 et N2 sont avant tout déterminées par la nature de leurs propriétés primitives, qui conditionnent à leur tour l'impact de la suffixation.

Veritable se distingue par le caractère absolu de son emploi syntaxique, obligatoirement prénominal, et de par sa faculté à qualifier : . un substantif :

[5] This painting has become a veritable obsession and he has returned to it repeatedly over the past thirty years. BNC

[6] [... ] and meals are veritable dinner parties, served by candlelight using the Worthingtons' fine glass and silver ware. BNC

[7] If artists gather in stables, then New York in the summertime is a veritable art rodeo : group shows abound and it is a matchless time to take the measure of any given gallery. BNC

[8] They drove back to the house in total silence, Rory too confused and unhappy to utter a single word, knowing too well that if she did, the floodgates on her feelings would open, releasing a veritable tidal wave. BNC.

un groupe nominal complexe :

[9] A veritable flood of new locomotive liveries, some smart, some misguided and dreadful, transformed the station scene from a mass of standard blue and grey to an unpredictable display of sector showing off. BNC

[10] From 1860 on, however, a dramatic increase in the number of such works occurred; a veritable explosion of major publications took place which owed a great deal to the excitement aroused by The Origin of Species. BNC

[11] The vituperative messages which poured forth in a veritable torrent of abuse were repeatedly drowned by the dissenting majority who kept up an incessant chant : 'return to work'. BNC 
[12] The Master of the Horse controlled a dozen chief assistants who in turn were overseers of a veritable army of coachmen, grooms etc, responsible for the organization of horses and carriages. BNC

ainsi que des noms propres (ou à référent unique) :

[13] But to Paula Ladies'Fashions is a veritable Alladin's cave. BNC

[14] These were the high-class European courtisans [...] sensual, elegant, veritable Mata-Haris and Lola Monteses ... BNC

[15] She's a veritable Cassandra who sees doom and death in the flight of a sparrow. BNC

54 par son antéposition) que le référent possède des propriétés telles que N2, sur le plan sémantique, pourrait paraître inapproprié (ou en décalage) par rapport à la réalité extralinguistique. Bien que l'impact de la qualification varie selon la nature du syntagme nominal qualifié (simple, complexe, nom propre), la raison d'être de la modification adjectivale est ainsi toujours de justifier - par anticipation - la dénomination choisie (N2), et ce pour trois motifs possibles: soit parce qu'elle peut paraitre excessive ou hyperbolique (obsession, dinner parties), et on aura remarqué que le contexte-après (souligné en pointillé) légitime à son tour la désignation [5] [6], comme si l'énonciateur voulait prouver que le terme choisi (N2) est approprié; soit parce qu'elle est métaphorique, et que, en tant que telle, cette dénomination ne correspond pas à la réalité extralinguistique immédiate (art rodeo, tidal wave); en ce cas, une mention préalable (ici stables et floodgates) prépare le co-énonciateur - par frayage - à la métaphore finale, ce qui contribue à la cohésion discursive.

Il faut remarquer que lorsque l'adjectif se trouve en tête d'un groupe nominal complexe, le substantif qu'il modifie relève toujours d'une interprétation métaphorique ([9] [10] [11] 12]). Si l'on accorde le statut de modus à la dénomination métaphorique, on constate que le dictum (ce à quoi l'énonciateur renvoie « en réalité »), figure soit en amont soit en aval de la métaphore, ce qui correspond à deux stratégies énonciatives différentes. L'énonciateur peut choisir de nommer d'abord le référent au sens propre ( $a$ dramatic increase), puis procéder à la métaphore (a veritable explosion) : celle-ci traduit alors une reformulation en discours ([10] [11]). Dans le cas inverse, l'élaboration est préalable, et l'explicitation (postérieure) est tout entière destinée au co-énonciateur qui, à l'instant de la métaphore, ne dispose pas encore du système de références de l'énonciateur ([9] [12]), soit parce que N2 est un nom propre ou à référent unique (Aladdin's cave, Mata-Hari, Lola Montes, Cassandra), et que dans un tel emploi, il n'est pas auto-déterminé : il ne renvoie en effet pas au référent qu'il désigne. La qualification adjectivale n'est alors possible que moyennant une recatégorisation de fonctionnement : le substantif fonctionnant comme un dénombrable, est, de fait, créée une classe d'éléments possédant les propriétés prototypiques du référent originel, ce qui ouvre la voie à certaines opérations de détermination (extraction, renvoi à la classe), et de qualification (modification adjectivale). Du point de vue des propriétés de son référent, la dénomination (N2) est ainsi présentée comme tout à fait appropriée (veritable), mais les diverses opérations de détermination signalent qu'il s'agit là d'une antonomase ${ }^{16}$ : le référent ne doit être donc considéré que sous l'angle de ses propriétés, et non de son identité propre ; dénommer $\mathrm{X}$ par N2 équivaut (par métonymie) à lui en attribuer les propriétés, mais seulement les propriétés. Il n'y a aucune confusion possible sur l'identité de X, car l'antonomase maintient l'altérité. 

présupposant l'existence d'au moins deux éléments dans la classe; le fléchage (the), en vertu de sa valeur anaphorique, est donc exclu: le référent désigné par the veritable Cassandra coïnciderait avec celui désigné par le nom propre, et il n'y aurait pas antonomase. On constate ainsi que dans tous les cas de figure, veritable permet à l'énonciateur de modifier la valeur référentielle de $\mathrm{N} 2$ tout en affirmant que son référent en possède un trait prototypique (le nombre, l'abondance...) qui motive la dénomination imagée, a priori pourtant impropre au regard des référents décrits. Veritable (ce n'est pas un moindre paradoxe) vise ainsi à souligner que le référent de N2 n'est pas à ce à quoi sa dénomination renvoie au sens propre. Ce qualificatif, à lui seul, annonce et accrédite la métaphore. épithète, ce n'est pas parce qu'il dénoterait une propriété inhérente au référent de N2: car il ne confère aucune une propriété supplémentaire, et ne participe donc pas à la création d'une sous-classe. En outre sa suppression n'entraînerait pas de perte d'information sur le plan quantitatif.

Veritable marque en fait une opération de type qualitatif sur N2, par laquelle l'énonciateur confirme la parfaite adéquation de la dénomination métaphorique. On pourrait avancer l'hypothèse que si un adjectif ne constitue pas ce que l'on peut définir comme une propriété additionnelle objective (induisant la création d'une sous-classe) et qu'il est obligatoirement épithète, c'est parce qu'il justifie le dire (N2), et en ce sens aurait une valeur énonciative. C'est pourquoi la qualification serait syntaxiquement inséparable de la notion que l'énonciateur a, par métaphorisation, élaborée. À la différence de veritable, la transparence de la morphologie de marriageable et de treasonable contribue à investir ble d'un apport sémantique perceptible, comme l'illustrent ces gloses définitoires :

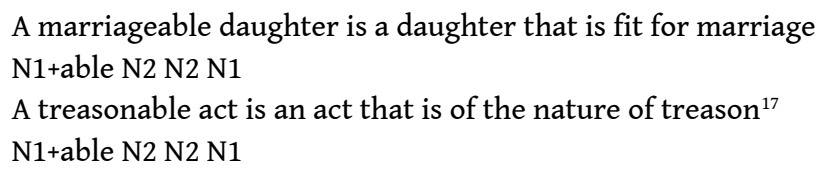

Quelle que soit la formulation (adj+nom/énoncé définitoire), c'est la même relation sousjacente qui unit les deux notions (N1 et N2). Mais sur le plan énonciatif, la stratégie diffère : lorsque la relation est établie par suffixation, ceci a pour effet de présenter la relation comme préconstruite, alors que la validation de daughter/be fit for marriage ou de act/be of the nature of treason équivaudrait à une caractérisation en discours.

Quelle relation le suffixe -able établit-il entre les deux notions ? La nature des référents de N2 étant différente, il importe d'analyser séparément marriageable et treasonable.

61 Marriageable La définition de OED est ambiguë, et contrairement aux apparences marriage étant un prédicat nominalisé - l'attribution de cette propriété n'est pas liée à la potentialité d'un événement (marry). Car si tel était le cas, marriageable correspondrait à la même réalité extralinguistique que unmarried (toute considération de différence de point de vue mise à part, donc sous l'angle du statut social). Mais marriageable n'équivaut pas nécessairement à unmarried :

[16] Coleridge himself cannot have failed to make the calculation that out of four marriageable Fricker sisters, one was married to Robert Lovell, and another all but engaged to Southey. BNC 
62 il serait pour le moins redondant de les associer au sein du même énoncé, à propos du
même référent:

[17] Farraline was, after all, of marriageable age and unmarried. BNC

Que de tels énoncés soient possibles met en évidence le rapport établi par -able entre les deux notions, et témoigne de la vraie nature de la qualification. Car N1, comme l'illustrent [16] et [17], n'évoque pas un procès auquel participerait N2 (en tant qu'agent ? ou patient ?). Puisqu'aucune occurrence de procès n'est en question, ce n'est pas sur le plan quantitatif que l'énonciateur se place, mais c'est à une évaluation de nature qualitative qu'il procède par cette qualification. Que cette attribution de propriété apparaisse sous forme adjectivale et en position épithète a pour effet de présenter cette propriété comme intrinsèque, mais elle n'est différentielle que du point de vue de l'énonciateur.

64 La même analyse vaut pour treasonable mais, puisqu'il ne peut qualifier un animé humain, la relation générée par la suffixation opère à un niveau différent.

65 La glose explicative met en évidence une relation d'équivalence entre N1 et N2 (an act that is of the nature of treason) : N2 est dit assimilable à N1. Les deux substantifs (prédicats nominalisés) renvoient en fait à un seul et même événement, et ceci est rendu possible par le sémantisme généraliste de N2, qui invite à la recharge sémantique. Cette recharge n'est pas de nature informationnelle, comme ce serait le cas lors d'une complémentation en of, qui, alors, définirait objectivement l'acte, et en discours. Car a treasonable act n'est pas nécessairement an act of treason. En tant que base de l'adjectif, treason ne renvoie pas (là non plus) à l'occurrence du procès en question : c'est act qui porte cette référence quantitative, tandis que treasonable a pour fonction de re-qualifier act, sous couvert de recharge sémantique. L'adjectif ne porte pas d'information sur l'événement lui-même, mais sur la perception que l'énonciateur en a : c'est son interprétation de la nature de l'acte qui est ainsi exposée.

66 Il est d'ailleurs significatif que treasonable ne fasse que très rarement l'objet d'une prédication directe en be, sans aucune modalisation, et c'est alors dans un énoncé à valeur définitoire :

[18] Harclay's motives were entirely understandable, but his action was technically treasonable, for he was 'a private person to whom it in no wise pertained to ordain such things'. BNC

67 Le plus souvent en effet, divers procédés modulent ou atténuent la valeur de vérité (fûtelle subjective) de la qualification (modalisation [19] [20], mention - en position initiale de la source énonciative [21]) :

[19] Many accepted the propaganda version of events, and even if they had begun to have doubts about Hitler, regarded the plot as a sacrilegeous and treasonable act against the head of State, and an attempted sabotage of the war effort. BNC

[20] In such an archetypal world, where 'good' is constantly and insecurely balanced in an eternal struggle against 'evil', the objective explication of the rituals and symbols which surround and mystify police work can seem tantamount to a treasonable act. BNC 
[21] In the eyes of the King of Morocco and the Moroccan authorities such ideas were treasonable. BNC

68 Compte tenu du fait qu'il ne s'agit là que de formes rares, peu usitées, que leur syntaxe n'est pas explicitement réputée contrainte (bien que les conditions d'emploi attestent d'une forte tendance à la position prénominale), nous conclurons cette analyse par ces quelques remarques.

Si l'origine dénominale de ces adjectifs apparaît comme un phénomène à l'impact secondaire, la suffixation s'avère en revanche une opération nécessaire lors de la mise en relation de semblables substantifs. Il ne serait en effet pas possible de former de tels mots composés ( ${ }^{*}$ marriage daughters, ${ }^{*}$ treason act).

En effet, selon Ruth Huart (1989), il existe cinq types de relations possibles entre les deux éléments constitutifs (A et B) d'un mot composé, compte tenu de leurs propriétés primitives :

- A et B peuvent être dans une relation d'identification (woman doctor)

- Ils peuvent faire l'objet d'une opération de différenciation (London buses - winter sports)

- A et B peuvent être dans une relation de dépendance (sand dune - eyelid)

- A et B peuvent constituer la source et le but d'une relation primitive (matchbox - teapot)

- $A$ et $B$ représentent deux des trois notions d'une lexis prédicable, la troisième présentant un caractère d'évidence tel qu'il est possible, sans dommage, de ne pas la nommer dans le mot composé (beesting - candle light)

$71 \mathrm{Si}$ *marriage daughters et *treason act ne sont pas des formations acceptables, c'est parce que leur relation sous-jacente ne s'apparente à aucun de ces schémas. Leur mise en relation ne peut donc passer que par la suffixation de N1 : seul -ble permet d'associer ces deux notions (N1 et $\mathrm{N} 2$ en surface), en les hiérarchisant. Ce que manifeste la suffixation, c'est qu'au terme d'une évaluation comparative des propriétés de N2 par rapport à celles de N1, l'énonciateur déclare N2 possesseur des propriétés attachées à N1. Puisqu'il ne s'agit pas de propriétés additionnelles, mais de caractéristiques considérées comme appartenant à N2, la qualification est de nature appréciative.

\subsubsection{Synthèse}

72 Il ressort de ces analyses que la suffixation en -able d'une base nominale est la trace d'une opération de repérage entre deux notions, l'une exprimée par la base de l'adjectif dérivé (N1 +-able), l'autre par le substantif qualifié (N2). Sous l'effet de la suffixation, les deux notions, associées, n'ont pas le même statut (ce que dénote leur appartenance finale à telle ou telle partie de discours), l'une (substantif) s'avérant être le support de l'autre (adjectif). Car dans cette relation, N2 constitue le terme repéré (c'est-à-dire, selon le niveau auquel on se place, la notion "principale ", le thème de discours, le noyau du syntagme nominal), N1 le terme repère (la notion "associée", occupant la place de déterminant dans le syntagme nominal) lui fournissant « une valeur référentielle qu'il ne possédait pas auparavant » (Culioli 1982)

Puisqu'il s'agit de formations anciennes (qui relèvent, conformément au schéma paraphrastique des dénominaux, d'une glose en that has the characteristics of), le repérage opéré par le suffixe est à valeur de différenciation : entre les deux notions est ainsi établie l'altérité, ce qui a pour effet de conférer au terme repéré (N2), les propriétés attachées au terme repère (N1) sans pour autant lui être identifiable :-ble bloquant ainsi l'entrée dans l'intérieur du domaine notionnel de $\mathrm{N} 1$, il y a donc ici construction d'une occurrence- 
frontière (Albrespit 1998). Le blocage résulte cependant de motifs dépendant de la nature du terme repère (et donc de ses propriétés).

Si ces propriétés sont attachées à la potentialité d'un procès, le blocage vient de ce que ce n'est pas à une validation éventuelle de ce procès que s'intéresse l'énonciateur. Il ne considère la notion que dans la perspective des propriétés physico-culturelles qu'il lui attache. (Déclarer qu'une femme a toutes les qualités d'une bonne épouse ne signifie pas qu'on envisage l'événement mariage).

Même si les propriétés de N1 sont, par nature, relatives à un procès, c'est seulement N2 qui, strictement parlant, renvoie à l'occurrence du procès (act). N1 (recharge sémantique) ne peut l'évoquer lui aussi : c'est un emploi qualitatif qu'il dénote. C'est pourquoi la suffixation n'établit pas une relation d'identification entre les deux notions (quand bien même elles renvoient au même événement). L'énonciateur déclare certes que $\mathrm{N} 2$ a les caractéristiques de N1, mais ne va pas jusqu'à affirmer que N2 est identique à N1 (dire qu'un acte a les caractéristiques d'un acte de trahison ne signifie pas, stricto sensu, qu'il y a eu trahison).

Si les propriétés attachées à N2, terme repéré (torrent) le sont au titre de la métaphore, le terme repère (Vveritas/) confirme l'existence d'un décalage délibéré et explicite entre la dénomination et la réalité extralinguistique. (Un torrent d'injures n'est pas un torrent).Dans chacun de ces cas, on constate ainsi comment le suffixe -ble marque la construction d'une frontière, et pour quelles raisons (différenciées, selon les configurations), le repérage qu'il opère bloque l'entrée dans l'intérieur du domaine de la notion exprimée par l'adjectif (simple évaluation comparative des propriétés, quasicorrespondance, métaphore).

\subsection{Adjectifs déverbaux}

On considère comme déverbal tout adjectif ${ }^{18}$ formé sur le radical d'un verbe ou bien sur sa racine participiale, qu'il s'agisse d'emprunts ou de créations, l'origine linguistique ne s'avérant avoir aucune incidence sur la syntaxe des adjectifs déverbaux du corpus.

Parmi les emprunts, presque tous viennent du français ${ }^{19}$, un seul du latin (inevitable).

Le français ayant hérité ces formes du latin, certaines constituent des dérivés opaques en anglais, soit parce qu'il n'existe pas de base verbale anglaise correspondant à la racine du dérivé (ar-able), ou bien parce que le sens de l'adjectif ne peut être déduit de ses composants apparents (respons-ible).

Les formations anglaises sont, conformément au schéma actuel, issues de verbes le plus souvent transitifs directs, mais aussi indirects (disposable), et sous la seule variante orthographique -able.

Deux sont des dérivés par préfixation : biodegradable, dishonourable.

Biodegradable (bio+degrad-able) est un dérivé savant, bio- constituant un préfixe extrêmement actif intervenant dans la création de nombreux néologismes, substantivaux (biohouse 1989 - biowashing powder 1989) ou adjectivaux (bioengineered cheese 1985).

L'origine de dishonourable est incertaine; selon OED, il est sans doute interprété comme dérivé de dishonour $\mathrm{vb}+-a b l e$, alors qu'il est plus probablement dérivé par préfixation (dis thonourable). 
83 Le préfixe bio- participe à la création d'un type (de dégradation). Son apport sémantique est fonction de la base: lorsque le dérivé n'est pas un terme scientifique, il évoque généralement une propriété « naturelle » (par opposition, selon les cas, à artificielle ou chimique).

Pour sa part, l'apport sémantique de dis- suggère l'opposé ou le négatif de l'adjectif non préfixé. Mais dishonourable n'est que partiellement l'antonyme de honourable: il ne qualifie en effet jamais directement un animé humain, seulement ses actes. Autrement dit, il est toujours associé à un prédicat nominalisé. Ceci par contraste avec honourable, qui ne connaît pas cette contrainte ${ }^{20}$. Cette différence montre que la préfixation peut restreindre l'emploi, et qu'une forme préfixée en dis- ne peut tout simplement être considérée comme « l'antonyme » de la forme simple.

\subsection{1. -ble témoin de préconstruction}

L'emploi de ces dérivés verbaux témoigne de deux opérations de préconstruction, l'une qui se manifeste sous forme lexicale (ces dérivés sont des adjectifs), la seconde par l'emploi syntaxique (ils sont employés en épithète).

86 La suite « adjectif+nom » résulte d'un schéma prédicatif dont la notion prédicative s'est réalisée sous la forme d'un adjectif (et non d'une forme verbale finie), et dont le syntagme nominal représente un des arguments (source ou but). L'adjectivation serait ainsi la trace d'une prédication antérieure (où l'activité aurait été prédiquée d'un sujet).

87 L'emploi épithète de ces dérivés verbaux serait la manifestation syntaxique de cette prédication antérieure : il est remarquable en effet que la position attribut, si elle n'est pas exclue, est cependant extrêmement rare. L'emploi en épithète peut alors être considéré comme une opération seconde.

\subsubsection{Nature des opérations sous-jacentes}

De quelles opérations le suffixe porte-t-il la trace ? Quelles relations instaure-t-il entre la base (la notion prédicative) et le substantif ainsi pré-modifié ?

À partir de cultivable land, et de sa glose définitoire (land that can be cultivated), on peut retracer la séquence des opérations en jeu.

On a au départ un schéma de lexis à trois places, l'une dévolue à la notion prédicative (/ cultivate/), les deux autres étant susceptibles d'être instanciées par les deux arguments de cette notion (la source et le but). Dans la suite adj+nom, seul un de ces arguments apparaît, et, comme c'est le cas ici, il s'agit le plus souvent de l'argument but : $<X-$ cultivate - land>. Le choix du terme de départ détermine l'orientation de la relation, et si le but est choisi comme thème de discours, l'orientation de la relation se traduit par la diathèse passive.

91 En outre, cette relation sujet/prédicat ne fait pas l'objet d'une validation (modalité de type 1), ce qui se traduirait par une assertion (land that is cultivated) mais d'une modalisation. En effet, « la validation de la relation passe par les propriétés du sujet de l'énoncé » (Bouscaren \& Chuquet 1987 : 37) : il y a assertion certes, mais de propriété. Le suffixe porte ainsi la marque d'un repérage d'ordre essentiellement qualitatif, comme en témoignent les gloses définitoires qui ne renseignent jamais sur une occurrence de procès. 
-Ble peut ainsi légitimement être considéré comme un marqueur de modalisation. Cependant, une analyse comparative des gloses des adjectifs du corpus montre qu'elles ne sont pas toutes réductibles à un seul schéma paraphrastique.

\subsubsection{1 -ble marqueur de modalisation radicale}

Dans de nombreux cas, le dérivé adjectival équivaut à une modalité de type radical, c'està-dire que l'on s'intéresse à la relation sujet/prédicat : l'adjectivation correspond alors à la mention d'une propriété de B. Mais cette qualification peut aussi prendre une valeur déontique lorsqu'y est associée une visée.

\section{Attribution de propriété}

94 L'association adj+nom ( $\mathrm{AB})$ peut se réécrire, comme dans l'exemple cité précédemment, en $B$ that can be Ved ( $V$ correspondant à la base verbale dérivante), ou it is possible for $B$ to be $V e d$, ou encore it is possible to $V B$. Relèvent de ce schéma les formations actuelles - $\mathrm{y}$ compris les néologismes - mais également des emprunts (arable/cultivable land), et des formations anglaises (drinkable water- recoverable resources - returnable bottles). Ces adjectifs sont issus de verbes transitifs, et $\mathrm{B}$ renvoie à un référent [-AH].

La modification adjectivale (A) équivaut à la mention d'une propriété (de B) qui a été construite antérieurement. En raison de son origine verbale, l'adjectif évoque un procès ( cultivate - drink - recover...), mais uniquement sur un plan virtuel : celui-ci peut en effet ne jamais se réaliser. Sa mise en œuvre dépend d'une intervention délibérée.

Dans le schéma de lexis, B correspond au but de cette notion prédicative. La relation prédicative est non saturée : la source est indéterminée - mais on sait qu'elle doit posséder (au moins) le trait [+animé] - et quelle qu'elle soit, la relation est validable. Par ailleurs, la glose ne se satisfait que d'une diathèse passive. Selon Gilbert (1987: 261), l'agent n'étant pas déterminé, seul le terme but peut constituer le repère prédicatif et constitutif de la relation prédicative, et ceci explique l'interprétation "possibilité » portée par can (et donc de -ble), l'ensemble permettant la construction de propriété du terme but.

97 La suffixation équivaut donc ici à une modalisation de type radical, et correspond à l'emploi prototypique de can: l'intervention énonciative est minimale, se limite à la mention d'une propriété de B. Avec ces adjectifs, la propriété est existentielle : elle vaut pour elle-même, dans le sens où elle n'est liée à aucune occurrence de procès.

Mais pour certains dérivés en -ble, l'existence de la propriété est indissociable du procès évoqué par l'adjectif. En effet, de façon très schématique, asserter l'existence de la propriété, c'est asserter la validation de la relation sujet/prédicat. En d'autres termes, dire que la propriété existe revient à dire que le procès se réalise.

Cet effet de sens est lié au sémantisme du verbe-base, au caractère naturel du procès (indépendant de toute intervention humaine). Celui-ci est inhérent au référent de N2; c'est une de ses propriétés physiques, constitutives, comme le sont la biodégradabilité d'un matériau, ou l'opposabilité du pouce chez certaines espèces :

[22] Composting is good, though highly labour-intensive because rubbish has to be sorted manually into biodegradable and non-biodegradable material. BNC

[23] Apes and monkeys, like humans, have an opposable thumb, which enables them to handle and investigate objects. BNC 

artefacts : validable) : (QLT).

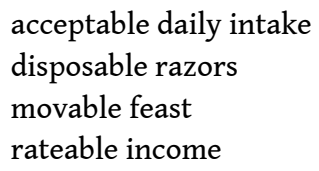

partie des propriétés de $B$, il n'est pas étonnant que propriété et procès soient ainsi confondus. Ce phénomène ne concerne pas seulement les propriétés physiques (naturelles), mais s'observe également à propos des propriétés culturelles attachées à des compte de la portée de la qualification; en effet, contrairement à ce que pourrait laisser croire le sens littéral de l'adjectif, le procès évoqué en A est bien validé (et pas seulement

- une dose journalière acceptable correspond à une dose effectivement acceptée

- un rasoir jetable se jette... après usage

- une fête mobile : sa date change effectivement (suite à une décision)

- un revenu imposable est le plus souvent imposé (au-delà d'une certaine limite, fixée antérieurement)

Il existe en fait toujours une étape préliminaire à la réalisation du procès. Le suffixe signale l'existence de cette condition préalable, dont l'évidence permet peut-être qu'elle ne soit pas explicitée.

On aura remarqué que si le procès est lié à une propriété physique, il n'est pas soumis à condition, et c'est parce que cette propriété est intrinsèque. Mais pour qu'une propriété culturelle soit pareillement liée à un procès, il est nécessaire d'y adjoindre une condition qui, une fois remplie, les associera tout aussi étroitement. La condition extérieure d'une propriété culturelle serait l'équivalent compensatoire du caractère intrinsèque de la propriété physique.

La fonction du suffixe est donc ici de mettre en relation une notion prédicative et un argument (but). De par leurs propriétés primitives respectives, il en résulte que possession de propriété équivaut à réalisation du procès. Le lien au procès est en effet très étroit: soit la propriété évoquée résulte d'un procès antérieur (acceptable daily intake, movable feast), ou bien elle est associée à un procès ultérieur dont la réalisation - même si elle est différée - est pour ainsi dire inéluctable (disposable razors, rateable income). Cependant, cette conséquence, secondaire, ne prive pas -ble de sa valeur fondamentale, car l'adjectif ne renvoie pas en lui-même à une occurrence de procès : c'est toujours et seulement dans la perspective de ses propriétés que la notion prédicative est évoquée

\section{Valeur déontique}

Le suffixe -ble peut établir une modalisation de type sujet/prédicat (valeur radicale) correspondant non à la construction d'une propriété comme dans les cas précédents, mais à la déclaration d'une nécessité, comme avec reportable.

Cet adjectif peut être considéré comme appartenant au vocabulaire technique : peu usitée , son emploi préférentiel en épithète participe à la création d'un type: sur le plan administratif, il existe deux types de maladie ou d'accident, ceux qui doivent faire l'objet d'une déclaration et les autres. 
[27] Questionnaires [...] show that only about a third of reportable accidents appear in official statistics. BNC propriétés extrinsèques de $B$ :

[24] Linda, Christy, Claudia, Naomi and Cindy are the world's most bankable beauties. BNC

Bankable signifie :

littéralement : that can be banked upon

en clair : certain to yield money sont pris en compte (yield money/ - /not yield money/), la valeur $\mathrm{p}$ est distinguée. La relation sujet/prédicat est en ce cas repérée par rapport à l'énonciateur et non plus par rapport à un autre agent potentiel (comme dans la valeur radicale). 
114 On notera aussi que la propriété syntaxiquement attribuée à B revient, sémantiquement, à l'énonciateur qui se prononce en fait sur les chances de validation du prédicat (yield money). Il s'agit là d'un cas d'hypallage syntaxique (John is sure to win) : l'adjectif informe sur le point de vue de l'énonciateur sur B (sur les chances de réalisation du procès), non sur B lui-même.

115 Inevitable, en position épithète, relève de la même analyse, son sémantisme se prêtant tout particulièrement à l'expression de la notion de prédiction :

[25] The Abbots united for the inevitable photograph bear more than a passing ressemblance to a sports team. BNC

116 En emploi attributif, inevitable peut, sans dommage, permuter avec sa glose (that can't be avoided) :

[26] He saw that if an electron could occupy any orbit round a nucleus then a spiralling collapse was inevitable. BNC

117 Le suffixe porte là une valeur radicale, et comme précédemment, il y a ici construction de propriété de l'argument but (a spiralling collapse); la source étant indéterminée, tout énonciateur ferait le même constat. N'est exprimée en ce cas aucune incertitude.

118 Alors qu'en épithète [25], si la source est effacée, elle est parfaitement identifiable : c'est l'énonciateur qui se prononce sur le caractère hautement prévisible de l'événement.

119 Pour résumer, lorsque l'adjectif est en position attribut, la prédiction se fonde sur les propriétés de B, évaluées dans la perspective de leur impact : l'événement est perçu comme une conséquence (hautement probable) des propriétés de B. Tandis que lorsque l'adjectif est prénominal, c'est à partir de ses propres connaissances que l'énonciateur déclare la haute probabilité du procès ${ }^{23}$. Par conséquent, selon que la source est déterminée ou non, l'adjectif renverra à l'expression du point de vue de l'énonciateur sur $B$ ou bien à la construction d'une propriété de B.Ainsi, selon que domine la valeur radicale ou épistémique, l'événement évoqué sera présenté soit sous l'angle de la potentialité, à propos du but (B) - et correspondra à l'attribution d'une propriété à $\mathrm{B}$ - soit de l'éventualité, c'est-à-dire du point de vue de l'énonciateur, et sans modification aucune des propriétés inhérentes de $B$.

\subsubsection{3 -ble marqueur de modalisation appréciative}

120 La qualification dont $\mathrm{B}$ fait l'objet peut correspondre à une attribution de propriété à valeur appréciative: l'adjectif informe alors sur le point de vue de l'énonciateur sur l'élément qualifié, dénote «l'appréciation favorable [...], défavorable, le jugement de normalité ou d'anormalité » (Groussier \& Rivière 1996: 18). La source est identifiable (même si elle n'est pas nommée) et c'est donc par rapport à l'énonciateur que le repérage est effectué.

Il est plus difficile qu'il n'y paraît de déterminer la nature appréciative d'un adjectif, en dehors des exemples prototypiques (bon, mauvais, beau, laid, agréable, souhaitable...). En l'occurrence, sera considéré comme appréciatif tout adjectif qui renseigne le coénonciateur non sur l'objet, mais sur le point de vue (en termes de jugement de valeur) de la source énonciative: autrement dit, un adjectif appréciatif n'est pas dédié à la construction de propriété, mais à l'expression du regard de l'énonciateur. 

responsible:

\section{eligible young man \\ honourable decision responsible citizen}

On notera en premier lieu que toutes constituent des emprunts, et qu'à l'exception de honourable, il n'est pas possible de formuler une glose paraphrastique faisant apparaître le verbe dont est dérivé l'adjectif. On s'appuiera donc sur les définitions dictionnairiques qui éclairent sur leurs affinités :

eligible : worthy of being chosen or elected honourable : worthy of being honoured responsible : trustworthy

L'étymologie de worth permet de comprendre la connotation appréciative de ces adjectifs. En vieil anglais, worth renvoie simplement au coût (pennyworth), puis au XIVe, il dénote la valeur en général (plus seulement monétaire) pour acquérir au XVI ${ }^{\mathrm{e}}$ le sens de valeur morale, intellectuelle, personnelle, d'où son acception actuelle de mérite.

L'interprétation de eligible et de responsible varie en fonction de leur emploi syntaxique. L'emploi attributif est toujours associé à une complémentation :

[27] Over 500,000 18-year-olds will be eligible to vote this year. BNC

La qualification signale que le sujet possède les propriétés requises pour valider le procès (contenu de la complémentation). L'emploi absolu est impossible, de même que pour

[28] These officials were responsible for the collection of revenue. BNC

Ainsi, l'adjectif attributif dit quelle relation existe entre le sujet (18-year-olds - these officials ) et le prédicat (vote this year - collection of revenue): il en explicite les conditions de réalisation en termes de propriétés du sujet (they can vote/ collect revenue). Dans ces emplois relatifs, la relation prédicative est saturée, la source et le but sont instanciés.

En position épithète, la qualification revêt une valeur appréciative :

[29] No matter how many eligible young men my uncle parades before me, I

shall never marry. BNC

[30] I thought it was a very responsible decision. BNC

29 Que l'emploi épithète (dans cette acception) soit obligatoire et non simplement possible ${ }^{24}$ montre bien que ce n'est pas à l'absence de complémentation que le qualificatif doit sa position prénominale. L'adjectif n'étant pas destiné à une fonction relationnelle (entre un sujet et un prédicat), son statut est différent : la propriété qu'il dénote est alors nommée pour elle-même. Et c'est par rapport à l'origine énonciative que la relation est repérée. La qualification traduisant ainsi le point de vue de l'énonciateur, elle est syntaxiquement inséparable du référent qualifié. 


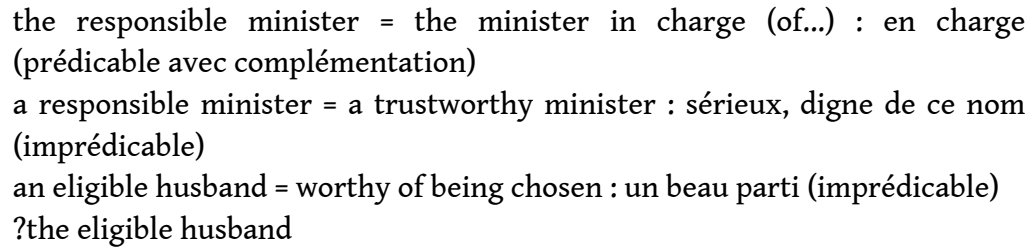

L'opération de détermination marquée par a porterait donc sur l'ensemble eligible husband ou responsible minister; en d'autres termes, il n'y aurait pas création (à l'aide de l'adjectivation) d'une sous-classe, mais extraction d'un élément de la classe (préexistante) eligible husband ou responsible minister, la propriété (eligible, responsible) étant posée comme constitutive de la notion (d'où l'emploi prénominal obligatoire).

Enfin on notera que la gradation n'est possible que lorsque l'adjectif a une valeur appréciative : a very responsible decision - one of the most eligible bachelors.

Si honourable est le plus souvent employé en épithète, c'est tout d'abord dans des formes d'adresse (honourable member), figées au point que l'adjectif est presque vide de sens ${ }^{25}$. Il peut cependant être réinvesti, mais on remarquera que c'est au prix d'un changement d'emploi syntaxique :

[31] As regards my right Hon. Friend's personal position, he's usually right and he is inevitably honourable. BNC

On s'intéressera donc aux emplois non figés, qui s'observent lorsque l'adjectif ne porte pas sur un animé humain. Rappelons que la valeur appréciative de honourable tient en premier lieu au sémantisme de sa racine (honour). Mais ce qui retient notre attention ici, c'est la modalisation qu'explicite la glose définitoire - worthy of being honoured - et dont on suppose que -ble est porteur. Dans les emplois suivants, cette glose ne parait cependant pas entièrement fidèle :

[32]... to encourage just and honourable practice. BNC

[33] Mulroney described the new constitutional agreement as a «fair and

honourable compromise that will strengthen Canada ». BNC

Plutôt que " digne de respect », il semble que l'énonciateur dit en fait « digne de ce nom », c'est-à-dire proper (également obligatoirement prénominal dans cette acception), en d'autres termes « qui correspond en tous points aux propriétés de la notion exprimée en B». La qualification signale que c'est au terme d'une évaluation comparative des propriétés de la notion et de l'objet de son analyse (B) que l'énonciateur parvient à cette conclusion. On est encore ici en présence d'une relation prédicative non saturée, mais la source est clairement identifiée, c'est par rapport à l'énonciateur qu'elle est repérée. De là honourable tire sa valeur appréciative.

L'analyse de ces dérivés a permis de mettre en évidence que leur emploi obligatoire ou préférentiel on épithète peut résulter de deux facteurs :

- soit la propriété dénotée par l'adjectif est considérée comme intrinsèque et l'emploi prénominal résulte d'une opération de prédication antérieure. Il est remarquable en outre que la qualification aboutisse à la création d'un type. Cette propriété peut être physique ( 
arable, biodegradable, cultivable, opposable), ou culturelle (acceptable, disposable, moveable, pensionable, rateable, reportable).

- soit la propriété est extrinsèque, et la position attributive est rare ou impossible. L'emploi en épithète manifeste que la relation prédicative est repérée par rapport à l'énonciateur qui présente ainsi la propriété comme, à ses yeux, caractéristique de B. Témoignent de l'expression du point de vue des adjectifs dénominaux (marriageable, treasonable, veritable), des dérivés à valeur épistémique (bankable, inevitable), ou appréciative (eligible, honourable, responsible).

137 Ainsi, dans l'un et l'autre cas - que la propriété soit intrinsèque ou non - la position prénominale est bien la marque d'une opération de préconstruction.

\section{Emploi attributif}

\subsection{Remarques générales}

Seuls neuf adjectifs sont soumis à cette contrainte. Pour certains, la nécessité de complémentation peut être considérée comme un facteur déclencheur :

[34] The Crown Estate is a public body accountable for itrs actions. BNC

[35] Most of the new agencies are answerable to the appropriate minister. BNC

[36] It is datable to the late twelfth century and attributable to the earliest named fabliau author, Jean Bodel. BNC

[37] Injury benefit is payable to anyone injured or disabled at work regardless of contributions. BNC

Il n'est pas possible de considérer incapable of et liable to sur le même plan car, suivis d'un verbe ou d'un prédicat nominalisé, ils constituent déjà en eux-mêmes une modalisation lexicale ${ }^{26} \mathrm{du}$ procès principal exprimé dans le groupe prépositionnel :

[38] Abandoned by her family and her servants, Elena Petrescu was incapable of grasping that a revolution had happened. BNC

[39]... Most sandgrouse are liable to flock with other species. BNC

La position attributive obligatoire peut être réservée à une acception spécifique : ainsi actionable, en épithète (place préférentielle pour ce sens), signifie « that can be done or used $»$ :

[40]... to explore actionable issues, products, technologies and trends in emerging application development areas .... BNC

mais en attribut, il appartient exclusivement au vocabulaire juridique « liable to an action at law»

[41] During the Republic, trusts were not actionable. BNC

La répartition des emplois syntaxiques correspond en ce cas à des acceptions différenciées. 


\subsection{Valeurs - Interprétations}

[42] It is, in effect, answerable to no-one although its members must of course answer to their own national governments. BNC

[43] A bill that is payable must be paid. LDOCE

Le suffixe peut également traduire une modalisation de type épistémique. L'énonciateur fait part de son incertitude quant à la réalisation de la relation prédicative :

[44] Other explanations of failure are more clearly attributable to donors. BNC

On pourrait arguer que le sémantisme de la racine dérivante (attribute) manifeste déjà une interprétation de la relation probable de cause à effet entre les deux éléments. En ce cas, attributable serait doublement épistémique: de par le sémantisme de la base d'une part, du fait de la dérivation d'autre part.

\subsection{Synthèse}

L'analyse de ces dérivés a établi que la suffixation a pour objet de conférer une propriété au syntagme nominal qualifié, ou de manifester le point de vue de l'énonciateur sur un objet ou un événement : quelle qu'en soit la valeur, il s'agit toujours d'une opération de modalisation qui, par nature, ne s'intéresse pas à la question de la validation. Par rapport à la réalité des faits, l'énonciateur reste toujours ainsi dans le domaine virtuel.

Mais il nous semble que ces adjectifs jouent un rôle dans la pragmatique du discours : ils permettent en effet à l'énonciateur de ne pas procéder ouvertement à une assertion au sens strict (dans le sens de validation de relation sujet/prédicat); il n'y a pas de déclaration sur la valeur de vérité de la relation.

On constate ainsi la distanciation que permet l'adjectivation par rapport à l'assertion : car ces adjectifs, sur le plan rhétorique, sont bien assimilables à des euphémismes. Advisable en est une illustration emblématique : 
[45] Wellington boots are advisable, but they were not absolutely necessary even in this exposed situation .... BNC

C'est bien entendu parce que advise est un verbe de dire que son dérivé adjectival fait du conseil que l'on pourrait prodiguer un conseil effectivement donné (valeur illocutoire ?). Available, dans cet emploi bien particulier, témoigne du même procédé :

[46] The President is not available for comment. LDOCE

Le président, dans les faits, se refuse à toute déclaration.

Et c'est pour ce même motif que attributable (également verbe de dire) peut, sans dommage, permuter avec attributed (avec une infime nuance sur le plan du contenu global du message). Il semble aussi fondé de considérer que accountable - ainsi que son synonyme answerable - et payable impliquent le même retrait par rapport à la réalité.

Sur la motivation de l'emploi syntaxique, on avancera qu'il est lié à cette présentation euphémistique - si l'on veut bien considérer que l'euphémisme manifeste la prise en compte du co-énonciateur - aux fins d'atténuer la portée d'une assertion. La prédication de la qualification en soulignerait - au niveau syntaxique - le caractère nouveau, inattendu, ou en l'occurrence décalé (par rapport à la réalité), en termes de point de vue évidemment.

\section{Conclusion}

L'objectif de cette étude était d'évaluer les impacts sémantique et syntaxique de la suffixation dans les dérivés en -ble. On a pu vérifier - et ceci confirme nos conclusions concernant d'autres adjectifs syntaxiquement contraints - que la position épithète vise à présenter la qualification comme préconstruite : soit du fait du caractère présenté comme intrinsèque de la propriété (physique ou culturelle), soit parce qu'elle témoigne d'un travail évaluatif antérieur de l'énonciateur. L'emploi attributif, qui correspond à la prédication d'une propriété en discours, témoigne pour sa part d'une authentique prise en compte du co-énonciateur : la qualification, ainsi mise en exergue de par sa position rhématique, porte une forte empreinte énonciative, et se signale (syntaxiquement) comme résultant d'une interprétation.

Le sémantisme de la racine participe pleinement de l'emploi syntaxique, et selon qu'il s'agit de verbes de dire ou de faire, la relation sous-jacente entre l'adjectif et le nom se traduit par divers schémas paraphrastiques dont le point d'ancrage est la présence d'une modalisation. Ceci est évidemment commun à tous les adjectifs en -ble, mais le choix d'un corpus fini nous a permis de circonscrire l'analyse et d'apporter des réponses concernant les contraintes syntaxiques. Le schéma dominant (mais non le seul) équivaut à une modalisation de type radical.

Il semble par conséquent que -ble doit être considéré comme un marqueur dont la valeur fondamentale est la modalisation : qu'elle soit de type radical, épistémique ou appréciatif dépend de la nature des éléments mis en relation, c'est-à-dire des rapports autorisés par leurs propriétés primitives.

Je remercie Pierre Cotte pour sa relecture attentive et ses suggestions. 


\section{BIBLIOGRAPHIE}

Albrespit, Jean. 1998. «Les adjectifs en -able ». Communication au $38^{\mathrm{e}}$ Congrès de la SAES.

Bauer, Laurie. 1983. English Word Formation. Cambridge : Cambridge University Press.

Bolinger, Dwight. 1967. «Adjectives in English. Attribution and predication ». Lingua 18, 1-34.

Bouscaren Janine et Jean Chuquet. 1987. Grammaire et textes anglais. Gap : Ophrys.

Bouscaren, Janine, Alain Deschamps et Catherine Mazodier. 1993. «Éléments pour une typologie

des procès ». Cahiers de recherche 6, Gap : Ophrys, 7-34.

Cotte, Pierre. 1996. L'explication grammaticale de textes anglais. Paris : Presses Universitaires de France.

Culioli, Antoine. 1982. « Rôle des représentations métalinguistiques en syntaxe ». $13^{\mathrm{e}}$ Congrès international des linguistes, Tokyo, DRL, Paris 7, 1-30.

Duchet, Jean-Louis et Jean-Michel Fournier. 1989. « Isomorphisme et productivité dans l'accentuation et la prononciation des mots dérivés anglais ». Le Suprasegmental II, 111-122, Paris : APLV.

Gilbert, Eric. 1993. « May, must, can et les opérations énonciatives ». Cahiers de Recherche en grammaire anglaise, tome 3. Paris : Ophrys.

Gilbert, Eric. 1998. «Quelques remarques sur as et la construction des valeurs référentielles ». Travaux linguistiques du Cerlico 11. Rennes : Presses Universitaires de Rennes, 103-126.

Groussier, Marie-Line et Claude Rivière. 1996. Les mots de la linguistique - Lexique de linguistique énonciative. Paris : Ophrys.

Guierre, Louis. 1979. « Essai sur l'accentuation en anglais contemporain ». Thèse d'état, Université Paris 7.

Huart-Friedlander, Ruth. 1989. « Nouveau regard sur les noms composés ». In A. Gauthier (dir.), Explorations en linguistique anglaise. Berne : Peter Lang.

Jespersen, Otto. 1914 - 1939. A Modern English Grammar on Historical Principles. Copenhague : Ejnar Munskgaard, Londres : Allen and Unwin.

Marchand, Hans. 1969. The Categories and Types of Present-Day English Word Formation. Munich : C.H. Beck'sche verlagbuchhandlung.

Merle, Gabriel et al. 1993. Néologie lexicale, anglais n5, GRIL, Université Paris 7.

Merle, Gabriel et al. 1996. Néologie lexicale, anglais n7, GRIL, Université Paris 7.

Schuwer, Martine. 1997. « Mots composés en V-ing + nom. Le cas remarquable des compositions elliptiques lexicalisées ». Travaux linguistiques du Cerlico 10. Rennes : Presses Universitaires de Rennes, 167-196.

Schuwer, Martine. 1999. "Étude sur les contraintes syntaxiques des adjectifs en -ed en anglais ». Cahier du CIEL 1998-1999, 110-151.

Tournier, Jean. 1985. Introduction descriptive à la lexicogénétique de l'anglais contemporain. Paris, Genève : Champion-Slatkine. 
Zandvoort, R. W. A Handbook of English Grammar, Londres : Longman, 1969.

\section{Dictionnaires}

Collins Cobuild English Language Dictionary. 1987. Londres : Collins.

The Concise Oxford Dictionary of English Etymology. 1986. Oxford : Oxford University Press.

Longman Dictionary of Contemporary English. 1987. Harlow : Longman.

Webster's New International Dictionary. 1968. Springfield, MA : Merriam.

\section{NOTES}

1. sur les formes en -ing (Schuwer 1997), sur les adjectifs en -ed (Schuwer 1999).

2. Ce développement est fondé sur Jespersen et Marchand.

3. Selon Duchet et Fournier, les adjectifs en -ible correspondent au schéma "pseudo-morphème +terminaison ", et devraient être considérées comme des unités "non dérivées ». Cependant, même si ces formations ne sont pas «reliées, pour le locuteur, à un dérivant par une nécessité sémantique claire ", la construction de la signification se fonde nécessairement sur l'association de ces deux éléments non autonomes. C'est pourquoi ils seront analysés au même titre que les autres adjectifs en -ble.

4. Ainsi, d'après OED, lovable (1340) serait dérivé de love (v)+-able. Mais selon Marchand, cette formation est assimilable à celle de comfortable : comfort (n)+-able.

5. Néologie lexicale 5 et 7

6. agreeable, assistable, comfortable, deceivable, (dis)cretable, durable, favourable, forcible, knowledgeable, perishable, risible, (un)shrinkable, (un)suitable, variable

7. knowledgeable (formé sur knowledge substantif) n'a pris son sens actuel qu'au XIX ${ }^{\mathrm{e}}$ (having knowledge); able illustre ici son étymologie latine : < habilis< habere (have)

8. slogan publicitaire, cité par Webster's

9. Webster's

10. Nous appliquons ici aux suffixes les critères de séparabilité des préfixes établis par Guierre.

11. La contrainte n'est pas toujours absolue et peut n'apparaître que dans certains emplois.

12. Par convention, N1 renvoie au substantif dont est dérivé l'adjectif en -ble, N2 au substantif qualifié.

13. British National Corpus

14. À titre comparatif, une recherche sur sensible aboutit à presque 3000 citations.

15. treacherous ou treasonous, eux aussi peu usités, seront alors utilisés.

16. du grec antonomazein : appeler d'un nom différent

17. gloses élaborées à partir des définitions données par $O E D$

18. ou dérivé par affixation d'un adjectif répondant aux mêmes critères de formation

19. acceptable - cultivable - eligible - honourable - miserable - movable - responsible - returnable. Un doute subsiste quant à l'origine latine ou française de arable.

20. Soit, en épithète d'un animé humain, il s'agit d'expressions rituelles, voire obligées ( $m y$ honourable friend, the Honourable MP), dont la fonction pragmatique atténue fortement l'apport sémantique.

21. BNC n'en donne que 10 occurrences en contexte (pour d'autres adjectifs, un minimum de 50 citations est proposé).

22. Il serait difficile de parler ici de "source ", dans la mesure où elle n'est pas véritablement agentive. La complémentation [28] est en effet introduite par under. C'est seulement le résultat du procès (les règles) qui est évoqué, et non l'événement lui-même (le moment où elles ont été établies). 
23. Il est d'ailleurs significatif que usual puisse ici facilement permuter avec inevitable : c'est bien sur ses connaissances que l'énonciateur s'appuie pour prédire l'événement.

24. On sait que, par nature, l'emploi épithète témoigne d'une opération de pré-construction. Mais ici, comme il est obligatoire, il ne peut ici être considéré comme consécutif à une prédication en be.

25. Comme en français, « cher collègue ", l'adjectif étant, là aussi, obligatoirement prénominal.

26. Il ne s'agit bien entendu pas d'une modalisation au sens strict, le sujet et prédicat n'étant pas mis en relation à l'aide d'un auxiliaire de modalité. Mais il y a bien assertion d'une propriété.

\section{RÉSUMÉS}

L'objectif de cette étude est de mesurer l'apport sémantique et l'impact syntaxique de la dérivation dans les adjectifs en -ble réputés syntaxiquement contraints, certains figurant obligatoirement en épithète, d'autres en attribut. On pose l'hypothèse que le comportement syntaxique différencié est lié à trois facteurs : au type de base suffixé, aux propriétés du référent que l'adjectif dérivé modifie; enfin à la nature de la relation que le suffixe établit entre la base dérivante et le nom-support.

This paper deals with syntactically constrained -ble adjectives, some being restricted to predicative position, others occurring attributively only: our objective is to assess both the semantic value and the syntactic impact of the suffix. The working hypothesis is that the syntax of such adjectives is linked to three factors, namely the nature of the stem (verbal/nominal), the properties of the noun modified by the derivative, and the relation established by the suffix between the stem and the noun.

\section{INDEX}

Mots-clés : adjectif, dérivé, modalisation, sémantique, syntaxe

Keywords : adjective, derivative, modalization, semantics, syntax

\section{AUTEUR}

\section{MARTINE SCHUWER}

Martine Schuwer est professeur de linguistique anglaise à l'université Rennes 2 Haute Bretagne. Ses domaines d'enseignement et de recherche sont la linguistique anglaise et la lexicologie.

Martine.Schuwer@uhb.fr 